\title{
Disfunción sexual y calidad de vida según el tipo de tratamiento renal sustitutivo
}

\author{
Patricia Ahís Tomás ${ }^{1,2}$, Elena María Renau Ortells ${ }^{2}$, Marta Meneu Oset ${ }^{1,}$ Vicente Cerrillo García ${ }^{2}$, Nayara \\ Panizo González ${ }^{1}$
}

${ }^{1}$ Centro de Hemodiálisis de La Vall de Uxó. ${ }^{2}$ Hospital General de Castellón. Castellón. España

\section{Resumen}

Se precisan estudios que evalúen el impacto de las distintas modalidades de tratamiento renal sustitutivo en referencia a su calidad de vida y disfunción sexual.

Objetivos: Evaluar la calidad de vida y la esfera sexual medidas por el cuestionario validado KDQOL-SFTM entre tres grupos de pacientes.

Material y métodos: Estudio observacional trasversal comparativo. Se estudiaron 72 pacientes $22,2 \%$ de peritoneal, $65,3 \%$ de hemodiálisis y $12,5 \%$ de domiciliaria.

Resultados: El 29,7\% de los pacientes en hemodiálisis consideran que la enfermedad renal crónica afectó "muchísimo" y "mucho" a su vida sexual. El 55,4\% de los pacientes en hemodiálisis admite problemas para disfrutar de la actividad sexual frente al $62,5 \%$ en peritoneal y $44,4 \%$ en domiciliaria.

Conclusiones: Los pacientes domiciliarios son los que presentan menor impacto sobre la esfera sexual.

\section{PALABRAS CLAVE}

- DISFUNCIÓN SEXUAL

- CALIDAD DE VIDA

- HEMODIÁLISIS

- DIÁLISIS PERITONEAL

- DIÁLISIS DOMICILIARIA

\section{Patricia Ahís Tomás}

Nefrovall, Centro de Hemodiálisis de La Vall de Uxó Polígono Industrial La Vernicha, 6

12600 Vall de Uxó. Castellón. España

E-mail: patriahis@gmail.com
Sexual dysfunction and quality of life depending on the type of renal replacement therapy

\section{Abstract}

Studies assessing the impact of different modalities of renal replacement therapy on patient's sexual dysfunction and quality of life are needed.

Objectives: To evaluate the differences in overall and sexual quality of life by validated questionnaire KDQOL-SF ${ }^{\mathrm{TM}}$ between patients receiving each renal replacement modality.

Methods: Observational comparative study. 72 total patients. Of them $22.2 \%$ were on peritoneal dialysis, $65.3 \%$ on hemodialysis and $12.5 \%$ on home hemodialysis.

Results: $29.7 \%$ of patients on hemodialysis consider chronic kidney disease affected "very much" and "a lot" their sexual life. $55.4 \%$ of patients on hemodialysis suffer from problems to enjoy sexual activity against $62.5 \%$ in peritoneal dyalisis and $44.4 \%$ at home hemodialysis.

Conclusions: Home treated patients are those with lower impact of the disease on its ability to maintain and enjoy sexual activity.

\section{KEYWORDS}

- SEXUAL DYSFUNCTION

- QUALITY OF LIFE

- HEMODIALYSIS

- PERITONEAL DIALYSIS

- HOME HEMODIALYSIS 


\section{Introducción}

La enfermedad renal crónica en su fase más avanzada, repercute en la función física y psicológica de las personas que la padecen ${ }^{1}$.

Las guías Kidney Disease Outcomes Quality Initiative (K/DOQI) sobre enfermedad renal crónica establecen que en el curso de esta se produce un deterioro de la calidad de vida relacionada con la salud de los pacientes, que está relacionada con factores demográficos (edad, sexo, nivel de educación, situación económica, etc.), con las complicaciones de la enfermedad renal crónica avanzada (anemia, malnutrición, etc.), con las enfermedades que la causan (hipertensión, diabetes, etc.) o con el propio deterioro de la función renal².

Todas las formas de terapia renal disponibles en la actualidad suponen un estrés para el paciente. El trasplante renal es la menos limitante al conllevar menores restricciones que la diálisis, menor dependencia (de la máquina, personal sanitario y de la familia) y mayor sensación de bienestar ${ }^{3}$.

En la National Institutes of Health Consensus Conference celebrada en 1993 en USA, fue definida la Disfunción Eréctil (DE) como la «incapacidad de lograr o mantener una erección suficiente para el desarrollo de una relación sexual satisfactoria» ${ }^{4}$. Las alteraciones de la esfera sexual abarcan también otros aspectos emocionales y físicos.

Las disfunciones sexuales contribuyen un problema que se manifiesta frecuentemente y que es necesario estudiar por la importancia que tiene para la salud de los individuos ${ }^{5}$.

Diversos estudios han puesto de manifiesto una elevada prevalencia de disfunción sexual entre los pacientes de ambos sexos portadores de una Enfermedad Renal Crónica (ERC) $)^{6-12}$.

Hay factores psicológicos, como la depresión y la ira, que actúan como indicadores de la etiología de las disfunciones sexuales. Las causas psicológicas más habituales son el estrés, la ansiedad, sentimientos de culpa, de inferioridad o baja autoestima y el temor a "no poder" en el momento del acto sexual, entre otras ${ }^{13,14}$.

En el caso de la mujer en diálisis puede haber descenso de estrógenos, amenorrea o hipomenorrea e incluso puede haber una disminución del tejido mamario, menor lubricación vaginal, vaginitis atrófica e infertilidad ${ }^{15}$.
Los trastornos hormonales en los hombres que están sometidos a tratamiento sustitutivo renal son atrofia testicular, disminución de la espermatogénesis y descenso de los niveles de testosterona y la presentación de impotencia parcial o total. En la fase urémica se manifiesta fundamentalmente como impotencia y disminución de la libido en el varón ${ }^{15}$. Juegan también un papel preponderante diversas toxinas urémicas, déficit de zinc y alteraciones hormonales tales como déficit de testosterona y elevación de procalcitonina y hormona luteínica que condicionan retraso en la espermatogénesis ${ }^{16-21}$.

La disfunción sexual se hace evidente en la fase urémica empeorando una vez iniciada la diálisis con disminución del deseo sexual. Existe una modificación de la corporalidad que limita el acercamiento físico con el otro, por la presencia del catéter de diálisis peritoneal y el aumento de volumen abdominal. Influyen también las alteraciones del aspecto físico como es la presencia de la fístula arterio-venosa en brazo, pérdida de masa muscular, desánimo e hipotensión después del procedimiento 22 .

La DE está relacionada con la edad pero no es exclusiva de la misma ya que el estudio Massachu et al, han demostrado la existencia de factores predictivos de DE como son la enfermedad coronaria, Hipertensión Arterial (HTA), Diabetes Mellitus (DM), dislipemia, tabaco, etc. La DM y la HTA son las causas principales de insuficiencia renal crónica terminal y la HTA es una constante en la evolución de las nefropatías así como en diálisis y en el trasplantado renal. La HTA condiciona DE por vía arterial y venosa al producir disbalance endotelial y disminución de producción de óxido nítrico. La DM es la enfermedad metabólica más frecuentemente asociada a DE y es sabida la íntima relación de la DM e HTA ${ }^{16-21}$.

La disfunción sexual constituye un problema sanitario de primer orden. Sin embargo, existen pocos datos en los que analicen la función sexual en estos tres tipos de técnicas, (diálisis peritoneal, hemodiálisis y diálisis domiciliaria). Por ello hemos decidido analizar la calidad de vida de los pacientes con problemas renales, y dentro de este indagar más sobre la sexualidad, ya que como se ha nombrado anteriormente es un problema a tener en cuenta en pacientes con esta patología.

Los objetivos del estudio son conocer la influencia de otras patologías en la disfunción sexual en pacientes con tratamiento renal sustitutivo y evaluar la alteración de la función sexual de los pacientes que reciben 
hemodiálisis (HD) en nuestra unidad y diálisis peritoneal (DP) y diálisis domiciliaria (HDD) en el Hospital General de Castellón mediante el cuestionario KDQOL-SFTM.

\section{Material y métodos}

Estudio observacional trasversal comparativo en pacientes con insuficiencia renal cónica, en las tres modalidades de tratamiento. La unidad de hemodiálisis del Centro de Nefrovall cuenta con 79 pacientes de los cuales 47 varones y 32 mujeres, con edades comprendidas 26 y 88 . La unidad de diálisis peritoneal del Hospital General de Castellón cuenta con 68 pacientes de los cuales 40 varones y 28 mujeres, con edades comprendidas 20 y 87 y por último los pacientes de domiciliaria de este mismo hospital son 9 de los cuales 5 varones y 4 mujeres, con edades 39 y 63 comprendidas. La muestra que accede y se somete a estudio es de 72 pacientes, 47 de HD, 16 de DP y 9 de HDD, previo consentimiento informado. Antes de iniciar la recogida de datos se informó verbalmente de la naturaleza del estudio asegurándoles la confidencialidad de los datos.

La recogida de datos se inició en el mes de enero y finalizó en marzo del 2016, en este periodo se entregó el cuestionario validado KDQOL-SFTM a los tres grupos. Elegimos este cuestionario porque posee una parte genérica y otra específica para pacientes en diálisis.

Los criterios de inclusión para el grupo de estudio son todos los pacientes que se encuentren es estas tres modalidades de tratamiento y que no cumplan los criterios de exclusión.

\section{Los criterios de exclusión son:}

- Aquellos pacientes que presenten alguna patología como la demencia que no puedan contestar correctamente a las preguntas.

- Negativa a participar en el estudio.

- Pacientes que no puedan leer o escribir por si solos.

Las variables demográficas añadidas en el estudio fueron: edad y género. Estas variables se obtuvieron en el cuestionario personal, sin tener que acceder a la historia clínica del paciente.

Las variables clínicas fueron: diabetes, hipertensión, hemoglobina, calidad de vida y disfunción sexual, las dos últimas se determinaron mediante el cuestionario validado KDQOL-SFTM.

El análisis estadístico se ha realizado en un paquete estadístico SSPS 18.0 para Windows. Para la relación entre variables cualitativas se empleó el estadístico de Chi cuadrado. Para variables cuantitativas se utilizó el test de Correlación de Pearson o el de Spearman, según los datos siguieron o no una distribución normal. Se aceptó significación estadística para $\mathrm{p}<0.05$.

\section{Resultados}

Un total de 72 pacientes cumplimentaron los cuestionarios en su totalidad, el $22,2 \%$ de peritoneal, $65,3 \%$ de hemodiálisis y $12,5 \%$ de domiciliaria, de estos $56,9 \%$ varones y $43,1 \%$ mujeres, con edades comprendidas entre 26 y 88 años. Las patologías que se tuvieron en cuenta fueron la diabetes y la hipertensión. No existen diferencias estadísticamente significativas entre la edad media, el sexo ni la proporción de hipertensos o diabéticos de los grupos.

Los pacientes en HD son los que se sienten más limitados para la realización de esfuerzos físicos intensos. A la pregunta " $¿$ Su salud actual le limita para esfuerzos intensos?" Contestaron "Si, mucho" un 65,3\% del total de pacientes estudiados, de los cuales el $74,5 \%$ eran pacientes en hemodiálisis en centro, frente a $56,2 \%$ de pacientes en DP y $33,3 \%$ de pacientes en $\operatorname{HDD}(p=0,019)$ (Figura I).

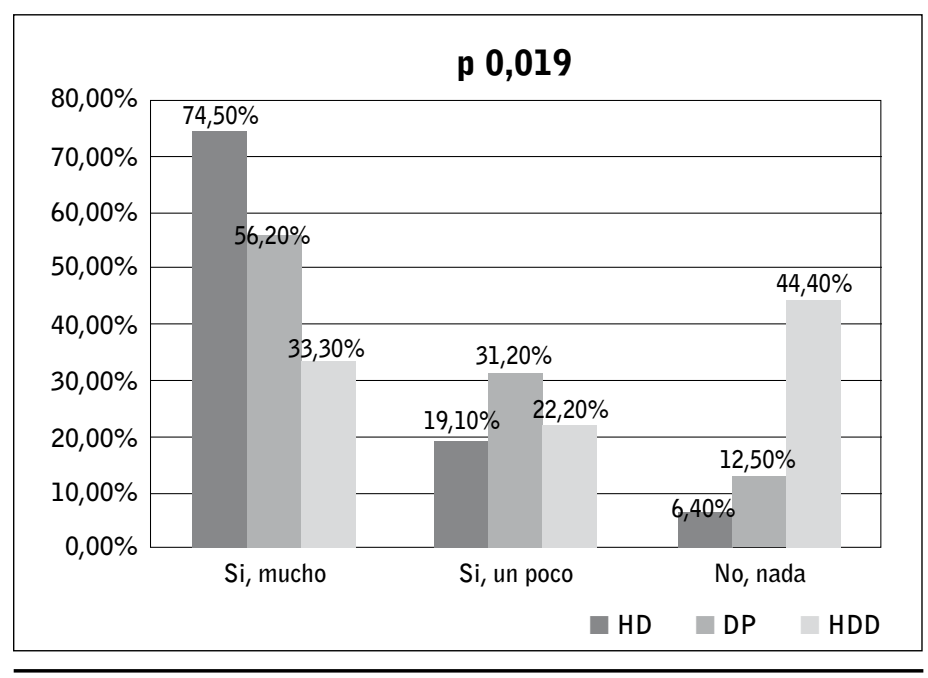

Figura I. Resultados a la pregunta: "Su salud actual, ¿le limita para hacer esfuerzos intensos, tales como correr, levantar objetos pesados, o participar en deportes agotadores?". 


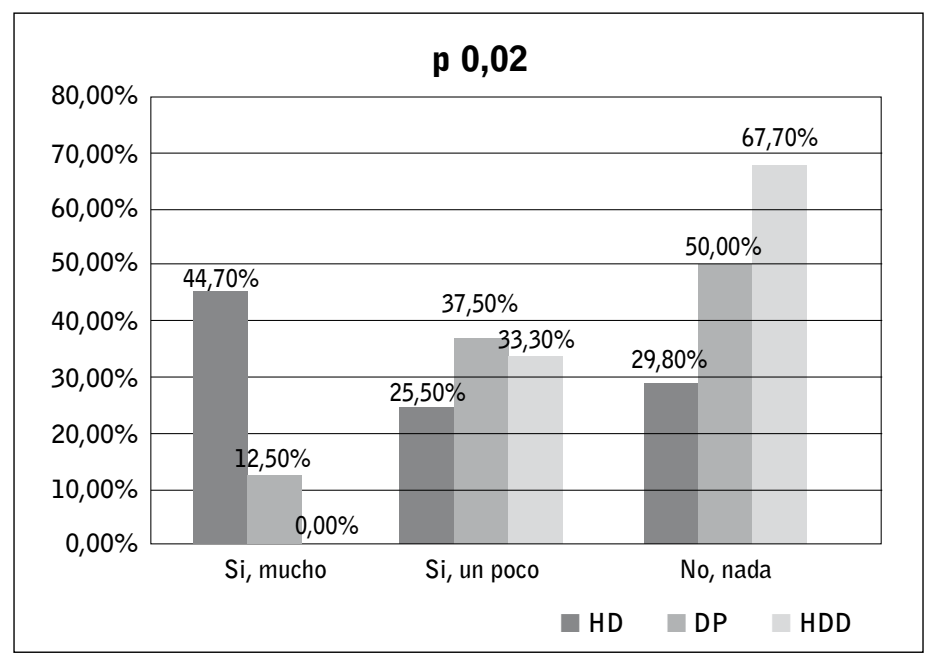

Figura II. Resultados a la pregunta: " Su salud actual, ¿le limita para hacer esfuerzos moderados, como mover una mesa, pasar la aspiradora, jugar a los bolos o caminar más de 1 hora?" .

En referencia a los esfuerzos de menor intensidad también se puede observar una diferencia estadísticamente significativa entre los tres grupos. Así, del $31,9 \%$ del total de pacientes que admite limitación importante para esfuerzos moderados, la mayoría son pacientes en hemodiálisis en centro $(44,7 \%)$, siendo mayor el porcentaje de pacientes en técnicas domiciliarias que no perciben dicha limitación $(p=0,02)$ (Figura II).

A la pregunta: "¿Cuánto tiempo se sintió tan bajo de moral que nada podía animarle?" el $33 \%$ de los pacientes en HDD respondió casi siempre frente a sólo el $4,3 \%$ de los pacientes en HD y ninguno de los pacientes en DP. Sin embargo, el $66,7 \%$ de los pacientes en HDD respondió "nunca" frente al $68,8 \%$ de los pacientes en DP y $53,2 \%$ de los pacientes en HD ( $p=$ 0,04) (Tabla I).

Tabla I. Respuestas a la pregunta: "Durante las 4 últimas semanas ¿cuánto tiempo Se sintió tan bajo de moral que nada podía animarle?".

\begin{tabular}{|l|c|c|c|}
\hline İTEM 9.C & Hemodiálisis & $\begin{array}{c}\text { Diálisis } \\
\text { peritoneal }\end{array}$ & $\begin{array}{c}\text { Diálisis } \\
\text { domiciliaria }\end{array}$ \\
\hline Siempre & $0 \%$ & $0 \%$ & $0 \%$ \\
\hline Casi siempre & $4,3 \%$ & $0 \%$ & $33,3 \%$ \\
\hline Muchas veces & $14,8 \%$ & $6,2 \%$ & $0 \%$ \\
\hline Algunas veces & $14,9 \%$ & $18,8 \%$ & $0 \%$ \\
\hline Solo algunas veces & $12,8 \%$ & $6,2 \%$ & $0 \%$ \\
\hline Nunca & $53,2 \%$ & $68,8 \%$ & $66,7 \%$ \\
\hline TOTAL & $100 \%$ & $100 \%$ & $100 \%$ \\
\hline
\end{tabular}

En las preguntas centradas en "su enfermedad del riñón", frente a un menor porcentaje en los grupos de DP $(62,6 \%)$ y HDD $(44,4 \%)$, encontramos un $89,4 \%$ de pacientes en hemodiálisis en centro entre los que consideran que la opción "su enfermedad interfiere demasiado en su vida" es "totalmente/bastante cier$\mathrm{ta}^{\prime \prime}(p=0,002)$ (Figura III).

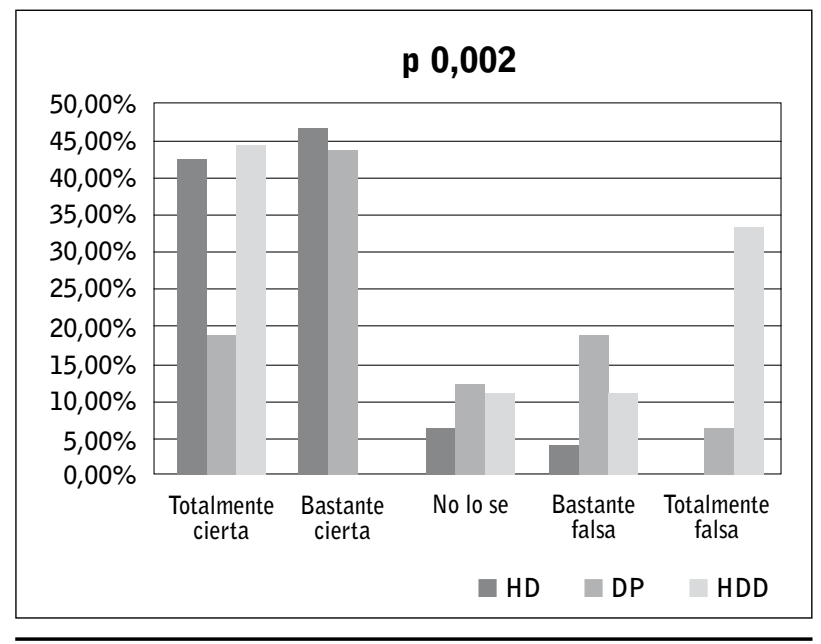

Figura III. Resultados a la pregunta: "¿En qué medida considera que su enfermedad del riñón interfiere demasiado en su vida?".

De entre los pacientes que consideran que "su enfermedad del riñón ocupa demasiado tiempo" también son mayoritarios los pacientes en hemodiálisis en centro, frente a un $50 \%$ de DP y un 55,5\% en HDD. ( $p=$ 0,011) (Figura IV).

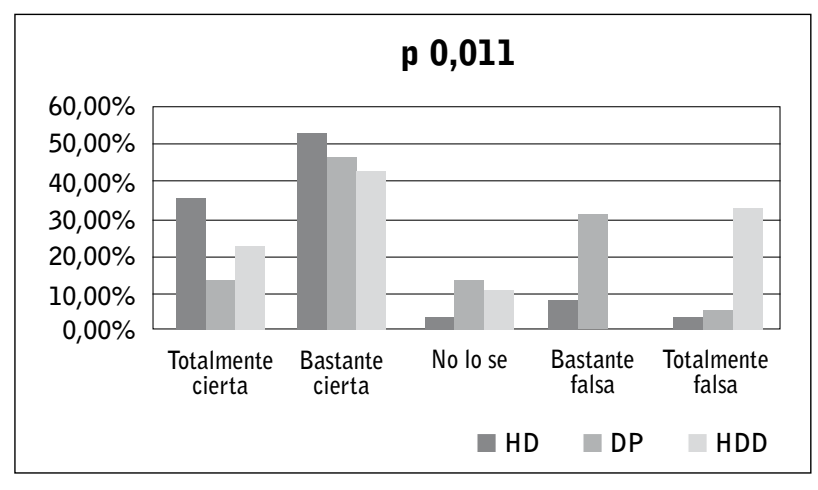

Figura IV. Resultados a la pregunta: "¿En qué medida considera que su enfermedad del riñón le ocupa demasiado tiempo?".

El $29,7 \%$ de los pacientes en HD consideran que la ERC afectó "muchísimo" y "mucho" a su vida sexual frente al $11,1 \%$ de los pacientes en HDD y $18,8 \%$ de los pacientes en DP. De entre los pacientes en DP las respuestas mayoritarias al grado de afectación de su vida sexual por la ERC fueron "un poco" 37,5\% 
y "regular" 37,5\%, entre los pacientes en HDD fue mayoritaria la opción "nada" 55,6\%, $(p=0,01)$ (Tabla II).

Tabla II. Respuestas a la pregunta: "¿Cuánto le molesta su enfermedad del riñón en cada una de las siguientes áreas? ¿Su vida sexual?"

\begin{tabular}{|l|c|c|c|}
\hline ÍTEM 15.G & Hemodiálisis & $\begin{array}{c}\text { Diálisis } \\
\text { peritoneal }\end{array}$ & $\begin{array}{c}\text { Diálisis } \\
\text { domiciliaria }\end{array}$ \\
\hline Nada & $44,7 \%$ & $6,2 \%$ & $55,6 \%$ \\
\hline Un poco & $12,8 \%$ & $37,5 \%$ & $33,3 \%$ \\
\hline Regular & $12,8 \%$ & $37,5 \%$ & $0 \%$ \\
\hline Mucho & $19,1 \%$ & $18,8 \%$ & $0 \%$ \\
\hline Muchísimo & $10,6 \%$ & $0 \%$ & $11,1 \%$ \\
\hline TOTAL & $100 \%$ & $100 \%$ & $100 \%$ \\
\hline
\end{tabular}

El $55,4 \%$ de los pacientes en HD admite problemas para disfrutar de la actividad sexual frente al $62,5 \%$ de los pacientes en DP y $44,4 \%$ de los pacientes en HDD, en cuanto a la respuesta mayoritaria a "ningún problema" se refleja en pacientes en HDD 55,6\%, frente al $36,2 \%$ en HD y $12,5 \%$ en DP $(p=0,04)$ (Tabla III). Sin embargo, no encontramos diferencias estadísticamente significativas en la capacidad para excitarse sexualmente.

Tabla III. Respuestas a la pregunta: " ¿Hasta qué punto supusieron un problema cada una de las siguientes cosas, durante las 4 últimas semanas?, Disfrutar de su actividad sexual"

\begin{tabular}{|l|c|c|c|}
\hline ÍTEM 16.A & Hemodiálisis & $\begin{array}{c}\text { Diálisis } \\
\text { peritoneal }\end{array}$ & $\begin{array}{c}\text { Diálisis } \\
\text { domiciliaria }\end{array}$ \\
\hline Ningún problema & $36,2 \%$ & $12,5 \%$ & $55,6 \%$ \\
\hline Muy poco problema & $8,5 \%$ & $25 \%$ & $0 \%$ \\
\hline Algún problema & $21,3 \%$ & $50 \%$ & $33,3 \%$ \\
\hline Mucho problema & $12,8 \%$ & $12,5 \%$ & $0 \%$ \\
\hline Muchísimo problema & $21,3 \%$ & $0 \%$ & $11,1 \%$ \\
\hline TOTAL & $100 \%$ & $100 \%$ & $100 \%$ \\
\hline
\end{tabular}

\section{Discusión}

Las disfunciones sexuales se consideran un tema sanitario de primer orden, porque pueden producir un deterioro potencial de la calidad de vida y del estado general de salud; en el estudio de Fernández (2002) manifiesta que el $75 \%$ de los pacientes en HD padecen $\mathrm{DE}^{23}$. Los pacientes en programa de HD de nuestro centro presentan una afección sexual menor a este estudio y a otros como el de Muñoz García (2010) ya que el $88 \%$ de los pacientes de este estudio lo expresa- ban $^{24}$. En la actualidad se considera la calidad de vida relacionada con la salud (CVRS) como uno de los objetivos terapéuticos primordiales en los pacientes que, por padecer una ECR, precisan tratamiento sustitutivo para seguir viviendo. Uno de los aspectos esenciales para alcanzar una buena CVRS es la posibilidad de mantener una actividad sexual satisfactoria.

Existen pocos estudios previos que comparen la prevalencia de algún tipo de disfunción sexual (disminución de la actividad y del deseo sexual) y calidad de vida con las diferentes modalidades de tratamiento sustitutivo renal (hemodiálisis, diálisis peritoneal y diálisis domiciliaria).

Nuestros resultados revelan mayor impacto físico de la enfermedad y su tratamiento con repercusión sobre la esfera sexual en los pacientes en hemodiálisis en centro, que también son los que sienten que la enfermedad interfiere más con su vida en general. Son varios los trabajos que previamente han constatado la mayor calidad de vida y autonomía en los pacientes sometidos a técnicas domiciliarias ${ }^{25}$.

En cuanto a la percepción de calidad como capacidad para disfrutar de la actividad sexual, el 55,4\% de los pacientes en HD refieren tener problemas. En estudios como Cordeiro y Lemos y Santos destacan la preocupación por las puntuaciones más altas en sus estudios $^{26,27}$. Es destacable, no obstante, que si bien son los pacientes en hemodiálisis los que consideran mayor el impacto de la enfermedad en su vida sexual, son los pacientes en diálisis peritoneal los que admiten en mayor porcentaje dificultades para disfrutar de la actividad $(62,5 \%)$.

A la hora de interpretar este resultado hay que tener en cuenta, además de la subjetividad, que el número de pacientes en tratamiento en DP incluidos en el estudio ha sido bajo, debido a que estos pacientes solo vienen a los controles bimensuales. En cualquier caso, consideramos que ser portador de un catéter de diálisis peritoneal y de líquido intraabdominal puede ser el principal condicionante de este resultado.

En nuestro estudio no hemos encontrado asociaciones entre la disfunción sexual y los problemas de salud relacionados con la HTA y DM. En diferentes estudios sí que aparece esta relación ya que reflejan que tanto la HTA como la DM son causas importantes para las disfunciones sexuales ${ }^{15,23}$. Hay estudios que reflejan que existe una relación en la edad y problemas sexuales ${ }^{28,29}$, no hemos encontrado esta asociación en nuestro 
trabajo, en posible relación con un alto porcentaje de pacientes con edad avanzada entre los participantes.

Debemos tener en cuenta como limitaciones a la hora de interpretar y comparar nuestros resultados y los de otros estudios, el carácter subjetivo de éste tipo de encuestas como limitación principal, así como la diferencia de tamaño muestral entre los grupos.

\section{Conclusión}

Al igual que en estudios previos, la prevalencia de algún tipo de disfunción sexual (afección sexual, problemas en su actividad sexual y excitación sexual) en nuestro estudio fue elevada.

Los pacientes en hemodiálisis en centros sanitarios son los que presentan mayor impacto de la enfermedad sobre su capacidad para mantener y disfrutar de la actividad sexual aun sin presentar mayor dificultad subjetiva para excitarse sexualmente. Este hecho parece tener un doble origen, derivado por un lado de una menor capacidad física, a mejorar con ajuste individualizado de la ultrafiltración y el control de anemia, y por otro del impacto emocional de la técnica en los individuos, mayor que en las técnicas domiciliarias.

Recibido: 25 octubre 2016

Revisado: 30 octubre 2016

Modificado: 7 noviembre 2016

Aceptado: 15 noviembre 2016

\section{Bibliografía}

1. Hays RD, Kallich JD, Mapes DL, Coons SJ, Carter WB: Developed of the Kidney Disease Quality of Life (KDQOLTM) instrument. Quality of life. Res. 1994; 3: 329-38.

2. World Health Organization. Quality of life assessment. An annotated bibliography. WHO. 1994 (MNH/PSF/94.1). Disponible en: https://books.google.es/books/content?id=pRwQA QAAM AAJ \&hl=es\&pg=RA1 PA24\&img $=1 \& p$ gis $=1 \& d q=1 . \% 09$ World + Health+Organization.+ Quality+of+life+assessment.+An+annotated+bi
bliography.+WH0.+1994+(MNH/PSF/94.1).\&sig=ACfU3U OrAm6X4YR2qwDRd9sWFnllzL$\mathrm{Jp} 3 \mathrm{~A} \&$ edge $=0$

3. De-Nour AK, Shannon J. Quality of life of dialysis and transplanted patients. Nephron.1980; 25: 11720.

4. National Institute of Health Consensus Development Panel on Impotence: Impotence. JAMA; 1993 270: 83.

5. Rubio E: Introducción al studio de la sexualidad humana. En: Conapo (ed.) Antología de la Sexualidad Humana. Miguel Angel Porrúa, I:17-46, México, 1994.

6. Levy NB: Sexual adjustment to maintenance hemodialysis and renal transplantation: national survey by questionnaire: preliminary report. 19: 138-43, Trans Am Soc Artif Intern Organs 1973.

7. Abram HS, Hester LR, Seridan WF, Epstein GM: Sexual functioning in patients with chronic renal failure. J Nerv Ment Dis.1975; 160: 220-6.

8. Diemont $W L$, Vruggink $P A$, Meuleman EJH, Doesburg WH, Lemmens WAJG, Berden JHM: Sexual dysfunction after renal replacement therapy. Am I Kidney Dis. 2000; 5:845-51.

9. Milde FK, Hart LK, Fearing MO: Sexuality and fertility concerns of dilysis patients. ANNA J 1996; 23: 307-313.

10. Palmer BF: Sexual dysfunction in uremia. J Am Soc Nephrol. 1999;10: 1381-8.

11. Berkman AH, Katz LA, Weissman R: Sexuality and the life- style of home dialysis patients. Arch Phys Med Rehabil. 1982; 63 272-5.

12. Toorians AW, Janssen $E$, Laan $E$, Gooren LJ, Giltay EJ, Oe PL, Donker AJ, Everaerd W: Chronic renal failure and sexual functioning. Clinical status objetively assessed sexual response. Nephrol Dial Transplant. 1997; 12: 2654-63.

13. Heiman J: Dysfunction: overview of prevalence, etiological factors and treatments. J Sex Research. 2002;1 (39): 73-78.

14. Sánchez Bravo C, Carreño Meléndez J, Martínez Ramírez S, Gómez López ME. Disfunciones sexuales femeninas y masculinas: comparación de género en una muestra de la Cuidad de México. Salud Mental 2005; 28 (4):74-80. 
15. Martín-Díaz F, Reig-Ferrer A, Ferrer-Cascales R. Función sexual y calidad de vida en pacientes varones de hemodiálisis. Nefrología 2006;26 (4):452-460.

16. Feldman HA, Goldstein I, Hatzichristou DG, Krane RJ, McKinlay JB. Impotence and its medical and psycho social correlates: results of the Massachusetts Male Aging Study. J Urol. 1994; 151: 54-61.

17. Burchardt M, Burchardt T, Baer L, Kiss AJ, Pawar RV, Shabsigh A. Hypertension is associated with severe erectile dysfunction. J Urol. 2000; 164: 1188-1191.

18. Kloner RA, Brown M, Prisant LM, Collins M.Effect of sildenafil in patients with erectile dysfunction taking antihypertensive therapy. AJH. 2001; 14: 70-73.

19. Toorians AW, Janssen E, Laan E, Gooren LJ, Giltay EJ, Oe PL Chronic renal failure and sexual functioning: clinical status versus objetively assessed sexual response. Nephrol Dial Transplant.1007;12: 2654-2663.

20. Palmer B: Sexual dysfunction in uremia. J Am Soc Nephrol. 1999; 10: 1381-1388.

21. Malavaud B, Rostaing $L$, Rischmann $P$, Sarramon JP, Durand D.: High prevalence of erectile dysfunction after renal transplantation. Transplantation. 2000; 69: 2121-2124.

22. Ramírez M. Insuficiencia renal crónica y vinculación comunitaria: representaciones sociales en usuarios en diálisis peritoneal. Rev Enfem Diál Tras 2012;13 (8):12-18.

23. Fernández Vega F. Disfunción eréctil en el paciente hipertenso y renal. Nefrología 2002; 22, supl 2 (12): 18-19.
24. Muñoz García VE, Vaca Ruíz AM, Romero EstudiIlo E, García Criado J, Bagdad Abselman A, Mohamed Maanan N. Comportamiento sexual en los pacientes de hemodiálisis en Melilla. Rev Soc Esp Enferm Nefrol. 2010;13 (2):113-118.

25. Walker RC, Hanson CS, Palmer SC, Howard K, Morton RL, Marshall MR, Tong A. Patient and caregiver perspectives on home hemodialysis: a systematic review. Am J Kidney Dis. 2015; 65(3):451-63.

26. Cordeiro JABL. Tratamento hemodialítico e qualidade de vida: avaliação do portador de insuficiência renal crônica. (Dissertação). Goiânia (G0): Faculdade de Enfermagem da Universidade Federal de Goiás; 2006.

27. Lemos VM, Santos OR. Disfunção sexual na insuficiência renal crônica sob hemodiálise regular / Sexual dysfunction in chronic kidney failure under maintenance hemodialysis. An Acad Nac Med. 1992; 152 (2): 8-13.

28. Martín F, Reig A, Sarró F, Ferrer R, Arenas D, González F, Gil T. Evaluación de la calidad de vida en pacientes de una unidad de hemodiálisis con el cuestionario Kidney Disease Quality of Life Short form (KDQOL SF). Rev Español. Diál. Tras.. 2004; 25(1):79-92.

29. Andreu Periz L, Force Sanmartin E. 500 cuestiones que plantea el cuidado del enfermo renal. $2^{\mathrm{a}}$ ed. Barcelona. Elsevier Masson; 2001. 